\title{
A Prospective Study to Evaluate Relationship between Lower Urinary Tract Symptoms and Erectile Dysfunction
}

\author{
Dr Nisar Ahmed ${ }^{1}$, Dr Lokesh Sharma ${ }^{2}$, Dr Vengetesh Kilvani Sengottayan ${ }^{3}$ \\ ${ }^{1,2}$ Department of Urology, NIMS Medical College, Jaipur, Rajasthan, India, 303121. \\ ${ }^{3}$ GKNM Hospital Coimbatore-641012
}

\begin{abstract}
Lower Urinary Tract Symptoms (LUTS) associated with Benign Prostatic Hyperplasia (BPH) and erectile dysfunctions $(E D)$ are highly prevalent in men aged more than 50 years and significantly increase with age and compromise quality of life.This study was aimed to determine the association and incidence of Lower urinary tract symptoms with erectile dysfunction and to examine the relationship between the different elements of LUTS (Storage and Voiding). This study was conducted in the Department of Urology, ChhatrapatiShahujiMaharaj Medical University, Lucknow, fromApril, 2009 - October 2010.Age group 61-70 has maximum no. of patients (39\%) enrolled with LUTS, Mean age of patients is 56.91 (Range 21-76). Severity of LUTS increases with age, $p=0.06$ which is close to being significant value. Odds Ratio of having IIEF-EF Score less than 22 in presence of specific frequency score of $\geq 3$ odds ratio is 2.294 and it is statistically significant $(p<0.05)$. We concluded that Mild to moderate erectile dysfunction is more prevalent in younger age group while severe erectile dysfunction is more prevalent in higher age group.
\end{abstract}

Keywords:ED, IIEF (Erectile Function) Score, LUTS,Storage IPSS sub score, Total IPSS score

\section{Introduction}

Lower Urinary Tract Symptoms (LUTS) associated with Benign Prostatic Hyperplasia (BPH) and erectile dysfunctions (ED) are highly prevalent in men aged > 50 years and both diseases significantly increase with age and frequently compromise quality of life. Erectile dysfunction (ED) has been defined as inability of a man to achieve, and/or sustain penile rigidity for sufficient time for a sexual performance. With aging, the prevalence of LUTS and ED increase in male to $31.2 \%$ and $52.1 \%$, respectively with resultant decrease in quality of life of the patients is a known fact $[1,2,3]$. In the past relationships between LUTS and ED was thought to be due to underlying prominent cause of aging but as the evidence has emerged and new insights are erupting about patho-physiology, this has recently generated great interest.

Several recent epidemiological studies assessed the association between LUTS and ED. Till date it is not known whether there is a common cause for ED and LUTS in some or all men with both problems. There is a biologically plausible link between ED and LUTS as suggested by several leading theories. It is yet to be determined how much the normal aging process can affect the patho-physiology of both ED and LUTS or these conditions influence each other.Increase in prevalence has been suggested to be related to multi-factorial conditions secondary to the aging process including metabolic syndrome (MetS), diabetes, and hypogonadism. In populationbased studies, a common patho-physiological basis for both LUTS and ED has been reported.

Many epidemiological studies have taken place all around the world including many Asian countries but yet to be done in Indian settings. In the present study we compare co-relationship between LUTS and associated erectile dysfunction.

Duration and place of study:

\section{Material And Methods}

This study was conducted in the Department of Urology, ChhatrapatiShahujiMaharaj Medical University, Lucknow, fromApril, 2009 - October 2010 according to working protocol.

\section{Inclusion Criteria:}

- All new patients presenting with history of Lower urinary tract symptoms with $>18$ yrs of age for $>3$ months duration with or without history of erectile dysfunction.

- Patient without any history of Medication for LUTS or Erectile dysfunction.

\section{Exclusion Criteria:}

- Patients with $<18$ yrs of age.

- History of - Bladder /urethral surgery

- Spinal surgery/spinal trauma 
- Neurological diseases

- Untreated and acute Urinary tract infection

- Patients with genitourinary tract or any other malignancy

- $\quad$ Patients on medical therapy for LUTS, ED and medications affecting libido.

- Patients with associated severe co-morbidities.

\section{Patient Work Up:}

Informed consent was taken from the patient before enrolling them for the study. All patients underwent detailed history along with filling of International Prostatic Symptom Score, International Index of Erectile Function Questionnaire Score, and physical examination.All patients completed the IPSS questionnaire including the question addressing quality of life .This was done in English/Hindi depending upon the patient preference. The IPSS was calculated by summing the scores of the 7 questions of the IPSS i.e. score on incomplete emptying (question 1), frequency (question 2), intermittency (question 3), urgency (question 4), weak urinary stream (question 5), straining (question 6) and nocturia (question 7). Score of each individual question ranged from 0-5. Patients were said to be having mild, moderate or severe symptoms when their total score was $0-7,8-19$ and 20 or more, respectively. The voiding sub score of IPSS were calculated by summing the scores of questions 1, 3, 5 and 6. The storage sub score of IPSS were calculated by summing the scores of questions 2,4 , and 7.

Male sexual function was also assessed using standardized, validated questionnaires. The IIEF is a 15item, standardized scale of male sexual function that assessed separate domains of erectile function (EF; six items), orgasmic function (OF; two items), sexual desire (SD; two items), intercourse satisfaction (IS; three items), and overall satisfaction (OS; two items). The IIEF is a gold standard measure of male sexual function, used in the majority of clinical trials in ED.The questionnaires consisted of Hindi versions of the IIEF-15. The gradual increase in the severity of ED were determined as per the age group (No ED: IIEF5 $\geq 22$; Mild: 17-21; Mild to moderate 12-16; Moderate: 8-11; Severe: $\leq 7$ ). This score was calculated by adding scores of first five questions and fifteenth question.LUTS and sexual function scores were calculated for the total sample and age cohort (21-30, 31-40, 41-50, 51-60, 61-70 and 71-80 years).

Laboratory Investigations and Radiological investigations (done with relevance to reach diagnosis of LUTS): Urinalysis (routine/microscopic), urine for $\mathrm{C} / \mathrm{S}$, routine blood investigations with serum creatinine, Ultrasound KUB-P (kidney, ureter, bladder and Prostate) region, Uroflowmetry with Post void Residual urine.

\section{Statistical analysis}

The data was described in terms of mean +/- standard deviation (SD) for continuous variables in the study population and in its subgroups. ANOVA (Analysis of variance) was done for Storage IPSS sub score, Voiding IPSS sub score, Total IPSS score and IIEF (Erectile Function) score between and within different Age Group.

Bivariate Pearson correlation coefficient after controlling for age was done between Storage IPSS sub score, Voiding IPSS sub score, Total IPSS score, IIEF (Erectile Function) Score, Intercourse Satisfaction, Orgasmic Function, Sexual Desire, Overall satisfaction and then finally all components of Storage IPSS sub score (Frequency, Urgency and Nocturia) and IIEF (Erectile Function) score using the Statistical Package for Social Sciences, version 16.0 (SPSS, Chicago, Ill) and the difference was considered to be significant if ' $p$ ' value was found to be $<0.05$.

\section{Results}

Table 1: Age wise distribution of Patients according to symptoms of LUTS

\begin{tabular}{|l|l|l|}
\hline Age group & Total No. of patients & Percentage of Patients \\
\hline $\mathbf{2 1 - 3 0}$ & 6 & 4 \\
\hline $\mathbf{3 1 - 4 0}$ & 11 & 7 \\
\hline $\mathbf{4 1 - 5 0}$ & 22 & 14 \\
\hline $\mathbf{5 1 - 6 0}$ & 47 & 30 \\
\hline $\mathbf{6 1 - 7 0}$ & 61 & 39 \\
\hline $\mathbf{7 1 - 8 0}$ & 9 & 6 \\
\hline Total & 156 & 100 \\
\hline
\end{tabular}

Age group 61-70 have maximum no. of patients (39\%) enrolled with LUTS followed age group 51-60 $(30 \%)$.Mean age of patients is 56.91 (Range 21-76). 
A Prospective Study To Evaluate Relationship Between Lower Urinary Tract Symptoms And...

Table 2: Age wise distribution of Patients according to severity of symptoms of LUTS

\begin{tabular}{|l|l|l|l|l|}
\hline Age groups & Mild (0-7) & Moderate (8-19) & Severe ( $\square$ 20) & Total \\
\hline $\mathbf{2 1 - 3 0}$ & $1(17)$ & $4(66)$ & $1(17)$ & 6 \\
\hline $\mathbf{3 1 - 4 0}$ & $2(18)$ & $5(46)$ & $4(36)$ & 11 \\
\hline $\mathbf{4 1 - 5 0}$ & $2(9)$ & $12(55)$ & $8(36)$ & 22 \\
\hline $\mathbf{5 1 - 6 0}$ & $5(10)$ & $21(45)$ & $21(45)$ & 47 \\
\hline $\mathbf{6 1 - 7 0}$ & $20(32)$ & $18(29)$ & $23(39)$ & 61 \\
\hline $\mathbf{7 1 - 8 0}$ & $0(0)$ & $6(67)$ & $3(33)$ & 9 \\
\hline Total & $\mathbf{3 0}(\mathbf{1 9} \%)$ & $\mathbf{6 6}(\mathbf{4 2} \%)$ & $\mathbf{6 0}(\mathbf{3 9 \%})$ & $\mathbf{1 5 6}$ \\
\hline
\end{tabular}

$\left(X^{2}=\mathbf{1 7 . 2 2}\right.$ and $\left.\mathbf{p}=\mathbf{0 . 0 6 9 6}\right)$

Severe LUTS is more prevalent in age group of 51-60 (45\%) followed by 61-70 group (39\%).Severity of LUTS increases with age, $\mathrm{p}=0.06$ which is close to being significant value.

Table 3: Age wise distribution of Patients with Severity of Erectile dysfunction (IIEF-EF Score)

\begin{tabular}{|l|l|l|l|l|l|}
\hline Age groups & Severe & Moderate & Mild to Moderate & Mild & No ED \\
\hline $\mathbf{2 1 - 3 0}$ & 0 & 1 & 0 & 2 & 3 \\
\hline $\mathbf{3 1 - 4 0}$ & 0 & 1 & 2 & 5 & 3 \\
\hline $\mathbf{4 1 - 5 0}$ & 0 & 1 & 1 & 9 & 11 \\
\hline $\mathbf{5 1 - 6 0}$ & 0 & 4 & 4 & 16 & 23 \\
\hline $\mathbf{6 1 - 7 0}$ & 3 & 3 & 4 & 26 & 25 \\
\hline $\mathbf{7 1 - 8 0}$ & 1 & 0 & 0 & 3 & 5 \\
\hline Total & $\mathbf{4 ( 2 \% )}$ & $\mathbf{1 0}(\mathbf{7 \%})$ & $\mathbf{1 1}(\mathbf{7} \%)$ & $\mathbf{6 1}(\mathbf{3 9 \% )}$ & $\mathbf{7 0}(\mathbf{4 5 \% )}$ \\
\hline
\end{tabular}

$\left(\mathrm{X}^{2}=\mathbf{1 3 . 0 0}\right.$ and $\left.\mathrm{p}=\mathbf{0 . 0 1 4}\right)$

Mild to moderate erectile dysfunction is more prevalent in younger age group while severe is more prevalent in higher age group.Severe to moderate ED is more prevalent in age group of 61-70 followed by 51-60 groups.

Table 4: Odds ratios for presence of ED (IIEF-EF <22) stratified by different aspects of LUTS.

\begin{tabular}{|c|c|c|c|}
\hline & Odds Ratio & $95 \%$ Confidence Interval & p Value \\
\hline \multicolumn{4}{|c|}{ IP55 (Total Score) } \\
\hline 0.7 & \multirow[b]{2}{*}{1.731} & \multirow{2}{*}{$.89-3.350$} & \multirow{2}{*}{0.1310} \\
\hline$>7$ & & & \\
\hline \multicolumn{4}{|c|}{ Frequeacy (Sub score) } \\
\hline 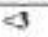 & \multirow[t]{2}{*}{2.294} & \multirow{2}{*}{$1.2-4.3$} & \multirow{2}{*}{0.0155} \\
\hline$\geq 3$ & & & \\
\hline
\end{tabular}

Odds Ratio of having IIEF-EF Score less than 22 in presence of specific frequency score of $\geq 3$ odds ratio is 2.294 and it is statistically significant $(\mathrm{p}<0.05)$.

Table 5: Correlation of IIEF - EF Score with patient response to IPSS questionnaires, age, prostate volume and uroflowmetry results (in 156 patients)

\begin{tabular}{|c|c|c|c|c|}
\hline Variable & Mean \pm SD & Range & $\begin{array}{l}\text { Correlation with } \\
\text { IIEF-EF (r) }\end{array}$ & $\begin{array}{l}p \\
\text { Value }\end{array}$ \\
\hline Age (in years) & $56.91 \pm 11.21$ & $21-76$ & -.051 & .529 \\
\hline IPSS total & $17.43 \pm 8.71$ & & .016 & .839 \\
\hline IPSS (Storage) & $7.18 \pm 3.91$ & $0-15$ & -.102 & .206 \\
\hline IPSS (Voiding) & $10.29 \pm 6.30$ & $0-20$ & .079 & .328 \\
\hline IIEF -EF Score & $20.16 \pm 4.96$ & $7-29$ & - & - \\
\hline $\mathrm{Q}_{\max }(\mathrm{ml} / \mathrm{sec})$ & $12.61 \pm 2.24$ & $5.7-19.9$ & .009 & .909 \\
\hline $\mathrm{Q}_{\max }(\mathrm{ml} / \mathrm{sec})$ & $8.78 \pm 6.2$ & $3.4-16$ & .025 & .758 \\
\hline $\begin{array}{l}\text { Postvoidresidual urine } \\
\text { Volume }(\mathrm{ml})\end{array}$ & $28.73 \pm 10.23$ & $0-170$ & .053 & .510 \\
\hline Prostate volume (ml) & $42.35 \pm 16.4$ & $12-88$ & .065 & 421 \\
\hline
\end{tabular}

Concise result of different parameters with IIEF -EF Score, age and IPSS (Storage) sub score are negatively correlated.

Table 6: Correlation between Storage IPSS sub score, Voiding IPSS sub score, Total IPSS score and Erectile Function

\begin{tabular}{|l|l|l|l|l|}
\hline \multicolumn{2}{|c|}{} & $\begin{array}{l}\text { Storage IPSS } \\
\text { sub score }\end{array}$ & $\begin{array}{l}\text { Voiding IPSS } \\
\text { sub score }\end{array}$ & $\begin{array}{l}\text { Total IPSS } \\
\text { score }\end{array}$ \\
\hline \multirow{2}{*}{ IIEF -EF Score } & Pearson Correlation (r) & -.102 & .079 & .016 \\
\cline { 2 - 5 } & $\begin{array}{l}\text { Sig. (2-tailed) } \\
\text { p Value }\end{array}$ & .206 & .328 & .839 \\
\hline
\end{tabular}

Correlation between Storage IPSS sub score, Voiding IPSS sub score, Total IPSS score and Erectile Function, on analysis we found that Strong negative correlation coefficient (r) exists between Storage IPSS sub 
score and Erectile Function implying inverse relation $(\mathrm{p}=0.206)$ but Correlation coefficient between Voiding IPSS sub score $(\mathrm{p}=0.32)$, Total IPSS score $(\mathrm{p}=0.83)$ and Erectile Function is not significant.

Table 7: Correlation between Storage IPSS sub score, Erectile Function, Intercourse Satisfaction, Orgasmic Function, Sexual Desire and Overall satisfaction

\begin{tabular}{|l|l|l|l|l|}
\hline & & Orgasmic Function & Sexual Desire & Orerall satisfaction \\
\hline \multirow{2}{*}{ IIEF-EF Score } & Pearson Contelation (r) & -478 & 371 & 166 \\
\cline { 2 - 5 } & Sig. (2-tailed) p value & 000 & 000 & .038 \\
\hline
\end{tabular}

Correlation between Storage IPSS sub score, Erectile Function, Intercourse Satisfaction, Orgasmic Function, Sexual Desire and Overall satisfaction, on analysis Strong negative correlation coefficient was present between Storage IPSS sub score and Erectile Function $(r=-.102, p=0.206)$ and intercourse satisfaction $(r=$ $-.179, \mathrm{p}=0.025)$.
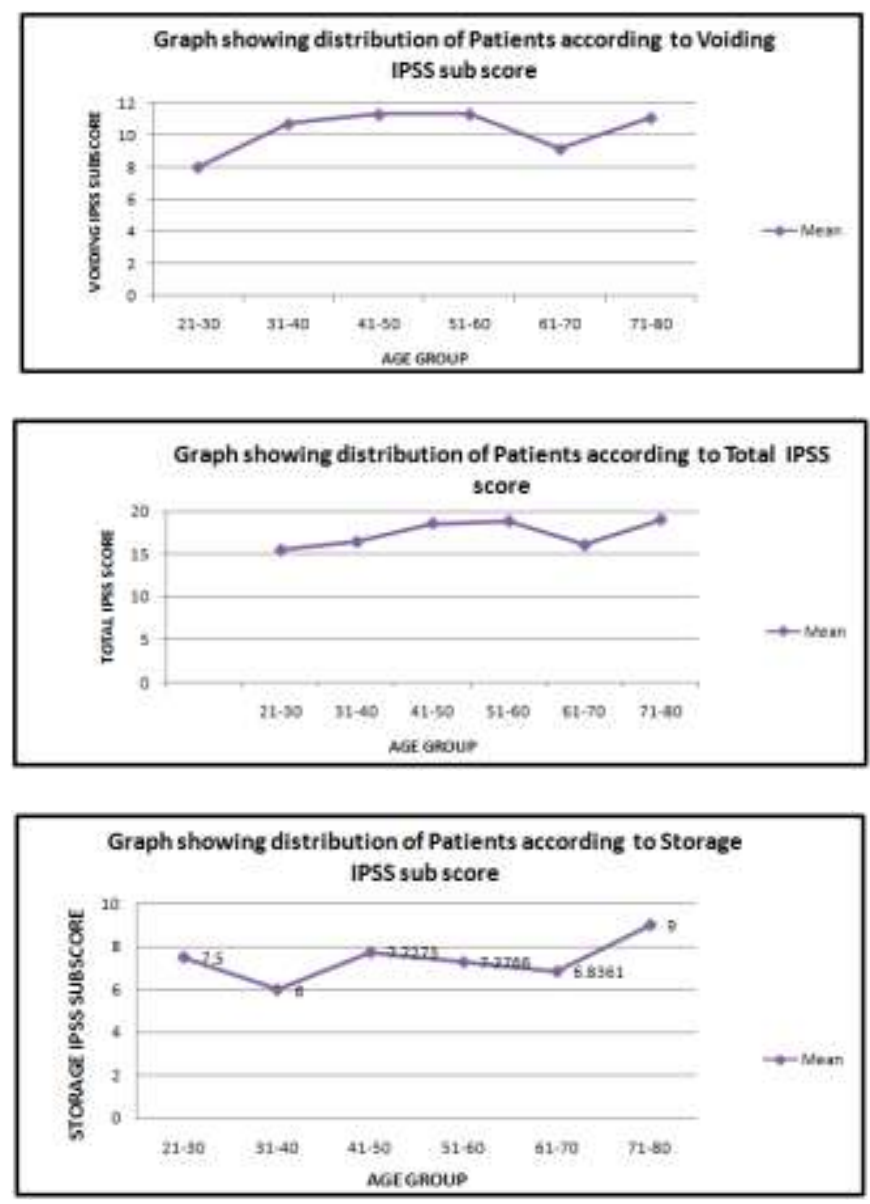

\section{Discussion}

The conventional opinion of no relationship between LUTS and sexual dysfunction was in the mind of urologists since long time, except for their presence more with ageing. Little evidence supporting the connection was available until the mid-1990s, when several epidemiological studies assessing the prevalence of BPH and associated quality of life issues suggested that LUTS by themselves could affect sexual function. Now, multiple studies have shown a strong association between these two entities, independent of other risk factors.Four mechanisms with varied degrees of overlap have been proposed:
a. Alteration in nitric oxide (NO) levels
b. Autonomic hyperactivity $(\mathrm{AH})$
c. Rho-kinase pathway
d. Pelvic atherosclerosis

Pharmacological agents prevalently used for the treatment of LUTS and ED have favorable effect; reflect the significance of these neuronal pathway in the patho-physiology.[4]LUTS consists of storage and 
voiding symptoms with dysfunction of bladder storage or emptying. In the age group of $>50$ years, BPH is a well-recognized clinical entity comprising enlargement of prostatic glandular tissue, concomitant narrowing of the urethra, with the subsequent development of LUTS.The incidence of BPH increases with age, as it is found in $50 \%$ of males over 50 years of age which increases to $90 \%$ in males over 80 years old, up to $50 \%$ of males with histology evidence of BPH experience LUTS, ED, or the inability to achieve, and/or sustain penile rigidity for sufficient time for a sexual performance, increases with aging.A rapidly increasing amount of epidemiologic data showing that LUTS and sexual dysfunction are inter-related clinical entities. The prevalence of LUTS in men with ED and without ED was $72.2 \%$ vs. $37.7 \%$ respectively, found in the Cologne Male Survey [5], and includes5000 German males of age group 30-80 years. Clinical trials have shown that Alpha-blocker agents and phosphodiesterase- 5 (PDE5)-inhibitor treatment results in significant improvement in LUTS. The exact mechanism of action is not well understood but most of studies do not demonstrated a significant effect of PDE5-I on peak flow $\left(\mathrm{Q}_{\max }\right)$.

The relationship between lower urinary tract symptoms (LUTS) and erectile dysfunction (ED) have received increased attention recently because high prevalence of both disease entities , more often both are seen frequently to occur in the same aging male group, and moreover contribute significantly to the overall quality of life.

Association between LUTS and ED is biased by the age factor which is found to be responsible. In the vast majority of guidelines and reviews on ED, LUTS was not considered to be an independent risk factor only. [6] Moreover as matter of fact, both disease entities are among the most frequent urologic disorders. But since then sufficient amount of evidence accumulated over the time, leading to establishment of causal relationship between the two coexisting entities. Since the mid-1990s, several epidemiological surveys have assessed the incidence and prevalence of BPH and associated quality-of life issues which suggested that LUTS by themselves could affect sexual function.

There is no such epidemiological surveys of any extent has been done to know burden of these two entities in India till today. Still we need to elucidate whether there is causal relationship between lower urinary tract symptoms (LUTS) and erectile dysfunction (ED) and if present, to how much extent they are related to each other.

The 2002 standardization committee report on terminology from the International Continence Society (ICS) [7] defined LUTS into three important categories: storage symptoms, voiding symptoms, and postmicturition symptoms. Storage symptoms having the components whichinclude nocturia, urgency, increased daytime frequency, urinary incontinence (stress urinary incontinence, urge urinary incontinence, mixed urinary incontinence, enuresis, nocturnal enuresis, continuous urinary incontinence, other different type of incontinence), bladder sensation (increased, reduced, normal, absent, non-specific). Voiding symptoms includes slow stream, intermittent stream, hesitancy, straining, splitting or spraying, and terminal dribble. Postmicturition symptoms indicate feeling of incomplete emptying and post-micturition dribble. Other related symptoms are pain symptoms of the genitourinary tract such as bladder, vulval, scrotal, perineal, urethral, and pelvic pain. AsLUTS can result from $\mathrm{BPH}$, it can also occur due to alterations inbladder function,neurologicaldisorders or other co-morbid conditions. Male LUTS and female LUTS are essentially the same; however, men are more likely to complain of voiding symptoms than women, and are less likely to experience urinary incontinence.

The causes of LUTS in middle-aged and older men are diverse, including lower urinary tract, prostate, nervous system, systemic diseases, and other pathological conditions. The most common causes would be benign prostatic hyperplasia, overactive bladder, underactive bladder, cerebrovascular disorder, polyuria, and their combination. In many instances, multiple causes are involved or no specific causes can be definitely identified.It is a well-recognized fact that LUTS are common in men age > 50 years [8] and are often caused by benign prostatic hyperplasia (BPH). [9, 10, 11]

ED, and LUTS are frequentcomplaints in patients with advanced age, and limited information is available for explaining the relationship between these two conditions exists.[2, 12, 13] Advanced age is an important factor; however some studies have demonstrated an association between BPH-related LUTS, and ED which is independent from advanced age. [4, 14].Association has not been statistically significant there were a clear negative correlation between Storage IPSS sub score and Erectile Function. $(\mathrm{p}=0.21)$ but Correlation coefficient between Voiding IPSS sub score $(p=0.32)$, Total IPSS score $(p=0.83)$ and Erectile Function is not significant.

There is a Strong negative correlation coefficient was present between Storage IPSS sub score with Erectile Function $(\mathrm{r}=-.102, \mathrm{p}=0.21)$ and intercourse satisfaction $(\mathrm{r}=-.179, \mathrm{p}=0.025)$ but storage IPSS score was not correlated significant to other variable like Orgasmic Function, Sexual Desire and Overall satisfaction. 
During analysis of multiplecorrelations between Total IPSS score, Voiding IPSS sub score, Erectile Function, Intercourse Satisfaction, Orgasmic Function, Sexual Desire and Overall satisfaction; we found that revealed that Correlation coefficient among them is not significant.

In this study when all components of Storage IPSS sub score and Erectile function were compared, we concluded that all components of Storage IPSS sub score (Frequency, Urgency and Nocturia) are negatively correlated $(\mathrm{r}=-.187, \mathrm{r}=-.029$ and $\mathrm{r}=.016$ respectively) with Erectile function but among components of Storage IPSS sub score, Frequency is most strongly showing Significance $(r=-.187, p=0.02)$.

In our study, Odds Ratio of having IIEF-EF Score less than 22 in presence of IPSS (Total Score) $>7$ is 1.731 but it is not statistically significant and in presence of specific frequency score of $\geq 3$ odds ratio is 2.294 , moreover association between them is statistically significant $(p<0.05)$.

When Multiple correlation between Storage IPSS sub score, Voiding IPSS sub score, Total IPSS score and Erectile Function was analyzed we found that there is Strong negative correlation coefficient present between Storage IPSS sub score and Erectile Function implying that if Storage IPSS sub score increases then Erectile Function score decreases $(p=0.21)$ but Correlation coefficient between Voiding IPSS sub score $(p=$ 0.32), Total IPSS score $(\mathrm{p}=0.83)$ and Erectile Function is not significant.

In our study when all components of Storage IPSS sub score and Erectile function were compared it revealed that all components of Storage IPSS sub score (Frequency, Urgency and Nocturia) are negatively correlated $(\mathrm{r}=-.187, \mathrm{r}=-.029$ and $\mathrm{r}=.016$ respectively) with Erectile function but among components of Storage IPSS sub score, Frequency is most strongly showing Significance $(r=-.187, p=-0.02)$ when correlated with Erectile function than Urgency or Nocturia.

In our study, Odds Ratio of having IIEF-EF Score less than 22 in presence of IPSS (Total Score) $>7$ is 1.731 but it is not statistically significant and in presence of specific frequency score of $\geq 3$ odds ratio is 2.294 , moreover association between them is statistically significant $(\mathrm{p}<0.05)$.

\section{Conclusion}

We concluded that Mild to moderate erectile dysfunction is more prevalent in younger age group while severe erectile dysfunction is more prevalent in higher age group. Now a days, the importance of quality of life in the elderly is rated very highly, but still recognition of sexuality with quality of life in old age is made a taboo subject especially in developing countries. It is now well recognized fact that approximately $50 \%$ of men aged over 60 possess sexual desire (libido). The creation of a taboo in this context is partly a consequence of the way the older generations were brought up, and is further promoted by the lack of attention paid to ED by even the medical profession. It will be necessary in future to investigate similar questions in women, so as ultimately to make it possible to draw some conclusions to guide health policy in relation to the quality of life of our older fellow-citizens, with special reference to their sexuality.

Reference

[1]. Bacon CG, Mittleman MA, Kawachi I, Giovannucci E, Glasser DB, Rimm EB. Sexual function in men olderthan 50 years of age: results from the Health Professionals Follow-UpStudy. Ann Intern Med 2003; 139:161-8.

[2]. Rosen R, Altwein J, Boyle P, Kirby RS, Lukacs B, Meuleman E, et al. Lower urinary tract symptom sandmalesexual dysfunction: the multinational survey of the aging male (MSAM-7). EurUrol 2003; 44:637-49.

[3]. Roberts RO, Jacobsen SJ, Rhodes T, Girman CJ, Guess HA, Lieber MM. Natural history of prostatism: impaired health states in menwith lower urinary tract symptoms. J Urol 1997; 157: 1711-7.

[4]. Mazur DJ, Helfand BT, McVary KT. Influences of neuroregulatory factors on the development of lower urinary tract symptoms/ benign prostatic hyperplasia and erectile dysfunction in aging men. UrolClin North Am 2012; 39:77-88.

[5]. Braun MH, Sommer F, Haupt G, Mathers MJ, Reifenrath B, Engelmann UH. Lower urinary tract symptoms and erectile dysfunction: co-morbidity or typical 'aging male' symptoms? Results of the 'Cologne Male Survey'. EurUrol 2003; 44: 588-594.

[6]. Lue TF. Erectile Dysfunction. N Engl J Med 2000; 342: 1802-1813. Wespes E, Amar E, Hatzichristou D, Hatzimouratidis K, Montorsi F, Pryor J et al. EAU guidelines on erectile dysfunction. EurUrol 2006; 49: 806-15.

[7]. Abrams P, Cardozo L, Fall M et al. The standardisation of terminology of lower urinary tract function: report from the standardization sub-committee of the International Continence Society. Neurourol. Urodyn. 2002; 21: 167-78.

[8]. Trueman P, Hood SC, Nayak US, Mrazek MF. Prevalence of lower urinary tract symptoms and self-reported diagnosed 'benign prostatic hyperplasia', and their effect on quality of life in a community based survey of men in the UK. BJU Int 1999; 83: 410-5.

[9]. Brookes ST, Donovan JL, Peters TJ, Abrams P, Neal DE. Sexual dysfunction in men after treatment for lower urinary tract symptoms: evidence from randomised controlled trial. BMJ 2002; 324: 1059-61.

[10]. Frankel SJ, Donovan JL, Peters TI et al. Sexual dysfunction in men with lower urinary tract symptoms. J ClinEpidemiol1998; 51: $677-85$.

[11]. Nickel JC. The use of alpha1- adrenoceptor antagonists in lower urinary tract symptoms: beyond benign prostatic hyperplasia. Urology 2003; 62 (Suppl. 3A): 34-41.

[12]. Ponholzer A, Temml C, Obermayr R, Madersbacher S. Association between lower urinary tract symptom sand erectile dysfunction Urology. 2004; 64:772-6.

[13]. Shiri R, Hakkinen JT, Hakama M, Huhtala H, Auvinen A, Tammela TL, et al. Effect of lower urinary tract symptoms on the incidence of erectile dysfunction. J Urol 2005; 174:205-9.

[14]. Mondul AM, Rimm EB, Giovannucci E, Glasser DB, Platz EA. A prospective study of lower urinary tract symptoms and erectile dysfunction. J Urol 2008; 179:2321-6. 